\title{
False vacuum decay in kink scattering
}

\author{
Adalto R. Gomes, ${ }^{a}$ F.C. Simas, ${ }^{b}$ K.Z. Nobrega ${ }^{c}$ and P.P. Avelino ${ }^{d, e, f}$ \\ ${ }^{a}$ Departamento de Física, Universidade Federal do Maranhão (UFMA), \\ Campus Universitário do Bacanga, 65085-580, São Luís, Maranhão, Brazil \\ ${ }^{b}$ Centro de Ciências Agrárias e Ambientais-CCAA, Universidade Federal do Maranhão (UFMA), \\ 65500-000, Chapadinha, Maranhão, Brazil \\ ${ }^{c}$ Departamento de Eletro-Eletrônica, \\ Instituto Federal de Educação, Ciência e Tecnologia do Maranhão (IFMA), \\ Campus Monte Castelo, 65030-005, São Luís, Maranhão, Brazil \\ ${ }^{d}$ Instituto de Astrofísica e Ciências do Espaço, Universidade do Porto, CAUP, \\ Rua das Estrelas, PT4150-762 Porto, Portugal \\ ${ }^{e}$ Centro de Astrofísica da Universidade do Porto, \\ Rua das Estrelas, PT4150-762 Porto, Portugal \\ ${ }^{f}$ Departamento de Física e Astronomia, Faculdade de Ciências, Universidade do Porto, \\ Rua do Campo Alegre 687, PT4169-007 Porto, Portugal \\ E-mail: argomes.ufma@gmail.com, simasfc@gmail.com, \\ bzuza1@yahoo.com.br, pedro.avelino@astro.up.pt
}

ABSTRACT: In this work we consider kink-antikink and antikink-kink collisions in a modified $\phi^{4}$ model with a false vacuum characterized by a dimensionless parameter $\epsilon$. The usual $\phi^{4}$ model is recovered for $\epsilon=0$. We investigate the $\epsilon \ll 1$ regime where the kink in the presence of false vacuum can be understood as a small deformation of the standard kink for the $\phi^{4}$ model. We show that the attractive interaction between the kink-antikink pair leads to a rich scattering pattern, in some cases delaying considerably the false vacuum decay.

KEywords: Solitons Monopoles and Instantons, Field Theories in Lower Dimensions, Nonperturbative Effects

ARXiv EPrint: 1805.00991 


\section{Contents}

1 Introduction 1

2 The model 2

3 Numerical results 3

3.1 False vacuum in an unbounded interval: kink-antikink collisions 3

$\begin{array}{ll}3.2 & \text { False vacuum in a bounded interval: antikink-kink collisions }\end{array}$

$\begin{array}{lll}4 & \text { Conclusions } & 10\end{array}$

\section{Introduction}

False vacua play an important role in fundamental physics, in particular in the context of inflationary models [1-3], electroweak phase transition and baryogenesis [4-8], bubble collisions [9-15], signals for the possible metastability of Higgs vacuum [16-18] and dark energy models $[19,20]$. Soliton solutions in nonlinear field theory describe localized energy concentrations which are able to propagate without modifying their shape. They have large applicability in condensed matter physics [21], optics [22], quantum field theory [23], nuclear physics [24-26] and cosmology [27-30]. The simplest soliton solutions are the kink and antikink in (1,1)-dimensions.

Kink scattering processes in nonintegrable models have intrincate structures. This has been studied not only in the simple $\phi^{4}$ model [31-40], but also in potentials of higher selfinteraction [41-48] and non-polynomial potentials [49-55]. Further interesting directions of investigation include the interaction of a kink with an impurity [56-58], kinks in models of two scalar fields [59-62], multi-kink collisions [63-65], boundary scattering [66, 67] and models with generalized dynamics [68].

Embedded in higher dimensional spaces, kinks give rise to domain walls and branes [69]. In some cyclic universe scenarios, domain walls were considered as a possibility to generate the Big Bang conditions [70-73], where planar symmetry is assumed and the dynamics is effectively $(1,1)$ dimensional. Solitons are also studied in the context of bubble collisions in the early universe. In the limit of a high nucleation rate per unit four-volume in comparison to $H^{4}$ (where $H$ is the Hubble expansion rate), one can neglect the spacial curvature of the universe and consider the bubbles as in flat spacetime [74]. In this regime, collisions of two bubbles have $\mathrm{SO}(2,1)$ symmetry and are described by a $(1,1)$ dimensional wave equation.

The mechanism of false vacuum decay in scalar field theories is well understood $[75,76]$. In particular, for an asymmetric double well potential, domain wall collisions with planar and $\mathrm{SO}(2,1)$ symmetry were considered in ref. [77]. Domain wall collision with such high degree of symmetry can be described as collisions between a kink and an antikink in $(1,1)$ 
dimensions. In this background the effects of small initial planar [77] and nonplanar [78] small fluctuations were investigated. However, ref. [77] only provided some examples for the output of background collisions, with no description of their dependence either with initial velocity or with the parameter controlling the difference in potential energy between the two wells.

The present work intends to fill this gap, with the aim of investigating the kink scattering and the corresponding false vacuum evolution, considering two situations: i) the false vacuum occupying the unbounded region outside the area defined by the kink-antikink pair; i) the false vacuum initially occupying the restricted region between the antikinkkink pair. We consider symmetric kink-antikink and antikink-kink collisions, with the pair initially at equal distances from the center of mass and initial velocities with same modulus. We show how the attractive interaction between the kink and antikink is the main factor affecting the structure of the scattering and, in some cases, delaying considerably the complete decay of the false vacuum even in a restricted region around the center of mass of the kink-antikink pair.

In the next section we review some of the main properties of the $\phi^{4}$ model. The numerical results are presented in the section 3 , and we conclude in the section 4 .

\section{The model}

We consider the action in $(1,1)$-dimensions in Minkowski spacetime with a Lagrangian with standard dynamics

$$
S=\int d t d x\left(\frac{1}{2} \partial_{\mu} \phi \partial^{\mu} \phi-V(\phi)\right)
$$

and a modified $\phi^{4}$ potential

$$
V(\phi)=\frac{\lambda}{4}\left(\phi^{2}-\frac{M^{2}}{\lambda}\right)^{2}-\frac{1}{2} \epsilon \frac{M^{3}}{\sqrt{\lambda}} \phi
$$

where $\epsilon$ is a dimensionless self-coupling parameter. From here on we will consider $\epsilon \geq 0$ without loosing generality. For $\epsilon \ll 1$ the potential has a local minimum (the false vacuum) $\phi_{+} \approx-M / \sqrt{\lambda}$, a local maximum at $\phi=0$ and a global minimum (the true vacuum) $\phi_{-} \approx M / \sqrt{\lambda}$. Here we are interested in the $|\epsilon| \ll 1$ limit, where the presence of the linear term in eq. (2.2) leads to a small force on the $\phi^{4}$ kink that favors the expansion of the true vacuum region [79]. For the sake of definiteness, we take $\lambda=M^{2}=2$. In this case the equation of motion is given by

$$
\ddot{\phi}-\phi^{\prime \prime}-2 \phi+2 \phi^{3}-\epsilon=0
$$

Static solutions can be found for $\epsilon=0$, given by the usual $\phi^{4}$ kink $\phi_{K}(x)=\tanh (x)$ and antikink $\phi_{\bar{K}}(x)=-\tanh (x)$. These solutions, of minimal energy, can also be obtained by first-order BPS equations [80, 81]. Applying a Lorentz boost, a travelling kink with velocity $v$ and initial position $x_{0}$ is obtained as $\phi_{K}(x, v, t)=\tanh \left(\gamma\left(x-x_{0}-v t\right)\right)$, with 
$\gamma=1 / \sqrt{1-v^{2}}$. Linear stability analysis around the static kink solution with $\phi(x, t)=$ $\phi_{K}(x)+\cos (\omega t) \eta(x, t)$ leads to a Schrödinger-like equation:

$$
-\eta^{\prime \prime}+\left(-2+6 \phi_{K}^{2}\right) \eta=\omega^{2} \eta
$$

This equation results in the eigenvalues [31] $\omega_{0}^{2}=0$ (the zero-mode or translational mode) and $\omega_{1}^{2}=3$ (the vibrational mode), followed by a continuum of states with $\omega_{k}^{2}=4+k^{2}$. The existence of vibrational modes is important for the comprehension of the complex behavior of kink-antikink scattering. One such effect is known as two-bounces, and it is characterized by a collision process where the initial translational energy is stored in the vibrational mode of the kink-antikink pair during an amount of time. As a result, the pair, after being scattered, oscillates around the contact point and retrocedes for a second collision. This mechanism was described in the ref. [33] as an exchange of energy between the translational and vibrational modes. Despite applicable in the present case, we remark that there are some known exceptions to this mechanism: in the $\phi^{6}$ model, two-bounces occur even in the absence of a vibrational mode [41]; the presence of more than one vibrational mode can result in the suppression of two-bounce windows [51]. Also, quasinormal modes can store energy during a collision in the $\phi^{4}$ model [40].

Now back to our problem for $\epsilon \neq 0$, as far as we know, there are no explicit solutions of the equation of motion. In particular, corrections $\phi_{n}$ to the kink field in $n$th order in $\epsilon$ were presented in ref. [79], and are described in terms of the basis of eigenfunctions $\left\{\eta_{n}\right\}$.

\section{Numerical results}

The kink scattering processes have a strong dependence on whether the initial region with false vacuum is finite or not. In the following we will consider separately kink-antikink and antikink-kink collisions.

\subsection{False vacuum in an unbounded interval: kink-antikink collisions}

First of all let us consider a symmetric kink-antinkink collision. We take as initial conditions a $\phi^{4}$ kink with velocity $v$ and a $\phi^{4}$ antikink with velocity $-v$ :

$$
\begin{aligned}
& \phi(x, 0)=\phi_{K}\left(x+x_{0}, v, 0\right)-\phi_{K}\left(x-x_{0},-v, 0\right)-1, \\
& \dot{\phi}(x, 0)=\dot{\phi}_{K}\left(x+x_{0}, v, 0\right)-\dot{\phi}_{K}\left(x-x_{0},-v, 0\right) .
\end{aligned}
$$

We fixed $x_{0}=15$ as the initial kink position, i.e., the kink solution centered at $-x_{0}$ and the antikink at $x_{0}$. Note that the initial condition is only a solution for $\epsilon=0$. We apply this even for $\epsilon \ll 1$ considering that the system will relax by emitting scalar radiation during their free propagation toward the collision region. To solve eq. (2.3) we use a pseudospectral method on a grid with 2048 nodes with periodic boundary conditions for $\phi$ and $\dot{\phi}$ and we set the grid boundary at $x_{\max }=200$. A sympletic method with the Dirichlet condition imposed at $x= \pm x_{\max }$ was also applied to double check our numerical results. We used a $4^{\text {th }}$ order finite-difference method with spatial step $\delta x=0.09$ and a $6^{\text {th }}$ order symplectic integrator with time step $\delta t=0.04$. 

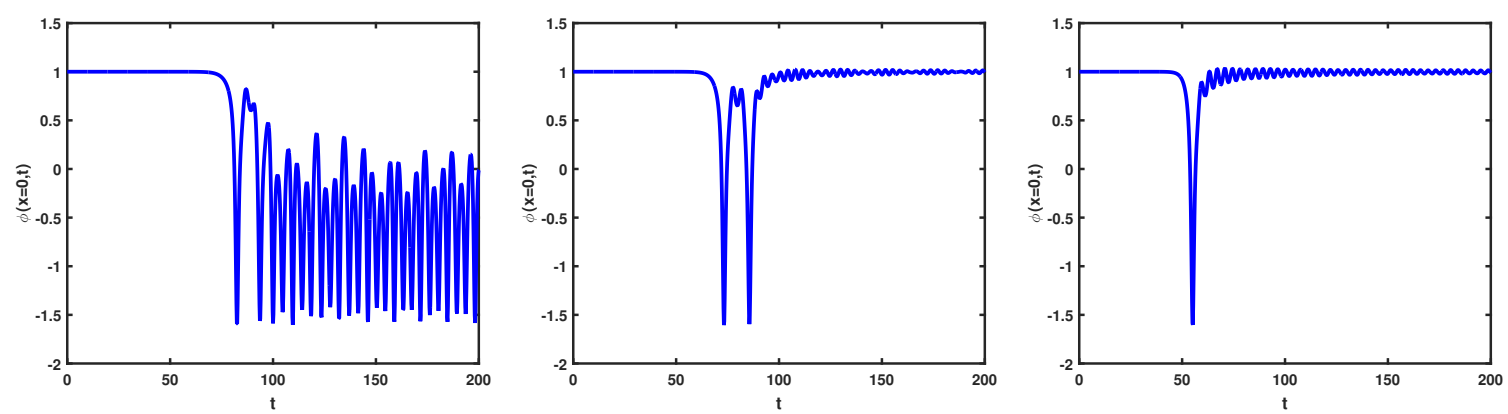

Figure 1. Kink-antikink collisions for the $\phi^{4}$ model, corresponding to $\epsilon=0$. Scalar field at the center of mass, $\phi(0, t)$, showing a) the formation of bion state (for $v=0.17$ ), b) two-bounce collision (for $v=0.1926$ ) and c) one-bounce collision (for $v=0.26$ ).

We start our analysis by reviewing some aspects of the $\epsilon=0$ case, corresponding to the $\phi^{4}$ theory, and well described in the literature [31-40]. Figures 1a-1c depict three profiles of scalar field at the center of mass as a function of time, $\phi(0, t)$, for different values of the initial velocity. Initially the point $x=0$ is at the vacuum $\phi=1$. For small velocities, the output of the scattering process is a bion state - see an example in figure 1a - where the pair radiates with the scalar field oscillating without a recognizable pattern until, in the long run, finishing at the vacuum $\phi=-1$. Velocities above a critical velocity $v_{\text {crit }} \sim 0.26$ show an inelastic scattering, where the output field retains the initial value $\phi=1$ after one bounce, as in the example of figure 1c. We could characterize this inelastic scattering as having $N_{b}=1$ bounces. For smaller velocities with $v \lesssim v_{\text {crit }}$ there are some velocity windows where the scalar field presents two-bounces $\left(N_{b}=2\right)$ during the collision process, as shown in figure $1 b$.

The structure of such two-bounce windows is better visualized in figure 2a, which shows the number of bounces as a function of the initial velocity. As shown in figure 1a, bion states have a large number of oscillations of $\phi(x, 0)$. Large values of $N_{b}$ are not directly represented in figure 2 , but there one represent bion states as making frontier with the two-bounce windows. Each two-bounce window is labeled by an integer $m$, the number of oscillations of $\phi(0, t)$ between the bounces. For instance, in figure $1 \mathrm{~b}$ we have $m=1$, meaning that this collision belongs to the first two-bounce window from figure 2a.

Now we turn to the effect of $\epsilon$ on kink scattering. Figures $2 \mathrm{~b}-2 \mathrm{~d}$ show the number of bounces as a function of the initial velocity for several values of $\epsilon \neq 0$. They show the inelastic scattering behavior with 1-bounce for $v>v_{\text {crit }}$, but with $v_{\text {crit }}$ growing with $\epsilon$. This is a direct effect of the tendency of the false vacuum to decay. One example of 1-bounce collision is presented in figure $3 \mathrm{a}$. We observed an effect that appears only for $\epsilon \neq 0$, that is the lack of bounces or even bion states for $v<v^{*}$ : the kink-antikink pair has not enought energy to encounter at the center of mass before the false vacuum decay. An example of this effect is shown in figure 3b. Also, 2-bounce windows are still present, but are thinner in velocity for larger values of $\epsilon$. One example of this is presented in the figure 3c.

The behavior of the kink-antikink pair after the collision process can be understood aproximating the static kink-antikink profile by

$$
\phi(x) \approx \phi_{K}(x+X)-\phi_{K}(x-X)-\frac{M}{\sqrt{\lambda}}
$$




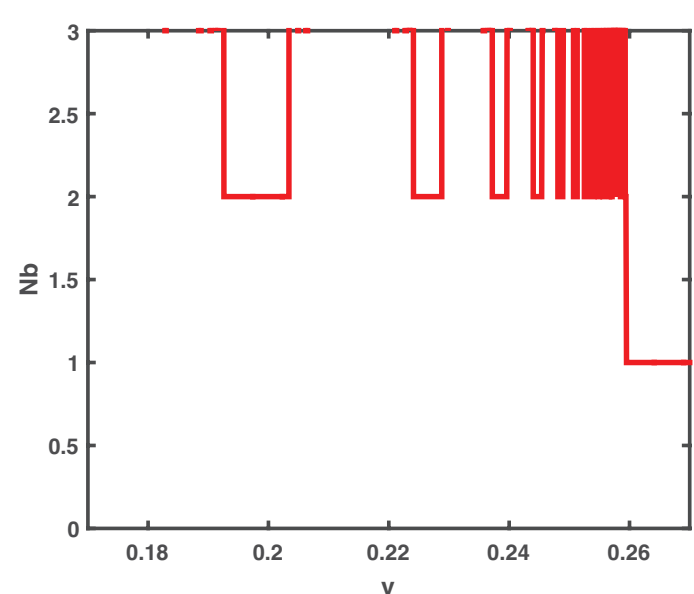

(a)

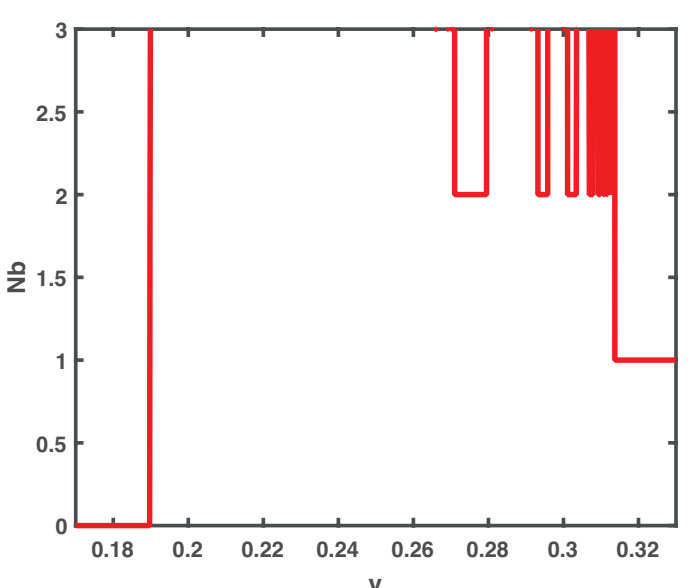

(b)

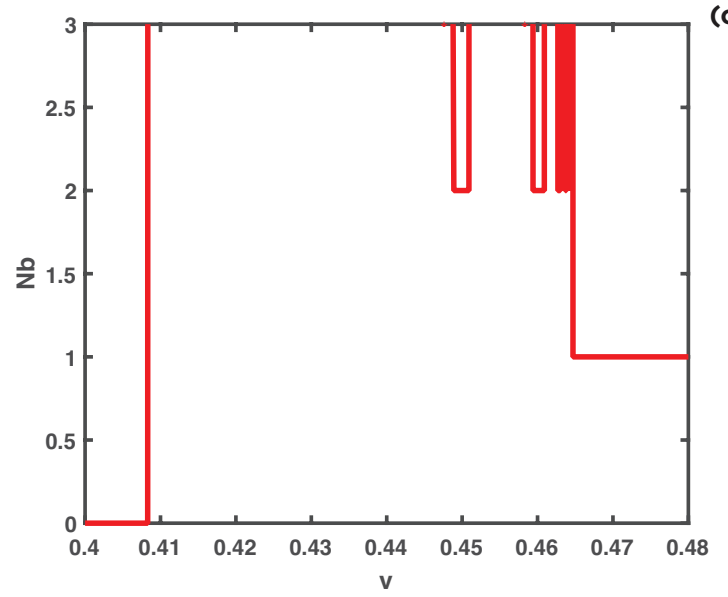

(c)

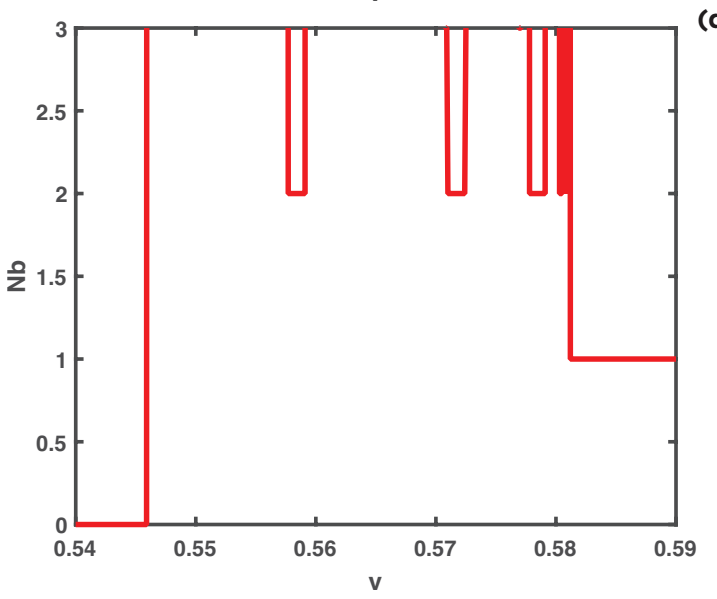

(d)

Figure 2. False vacuum in an unbounded interval: kink-antikink collisions. Number of bounces versus initial velocity $v$ for (a) $\epsilon=0,(\mathrm{~b}) \epsilon=0.001$, (c) $\epsilon=0.005$ and (d) $\epsilon=0.01$.
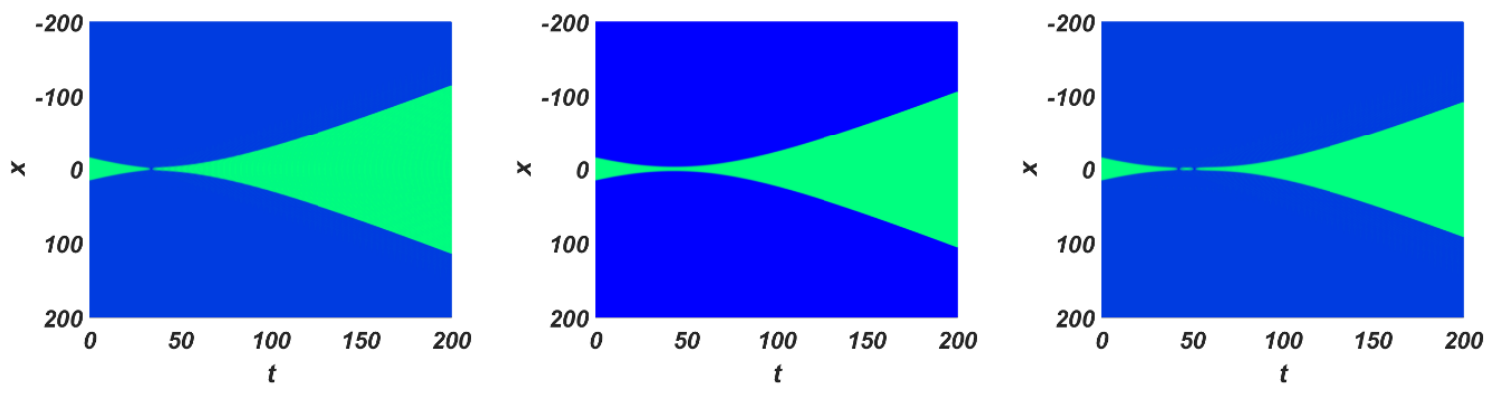

Figure 3. False vacuum in an unbounded interval: kink-antikink collision for $\epsilon=0.01$ with (a) $v=0.59$, (b) $v=0.54$ and (c) $v=0.558$. Compare with diagram of figure $2 \mathrm{~d}$. Blue represents the false vacuum $\phi=-1$ and the true vacuum $\phi=+1$ is represented in green. 


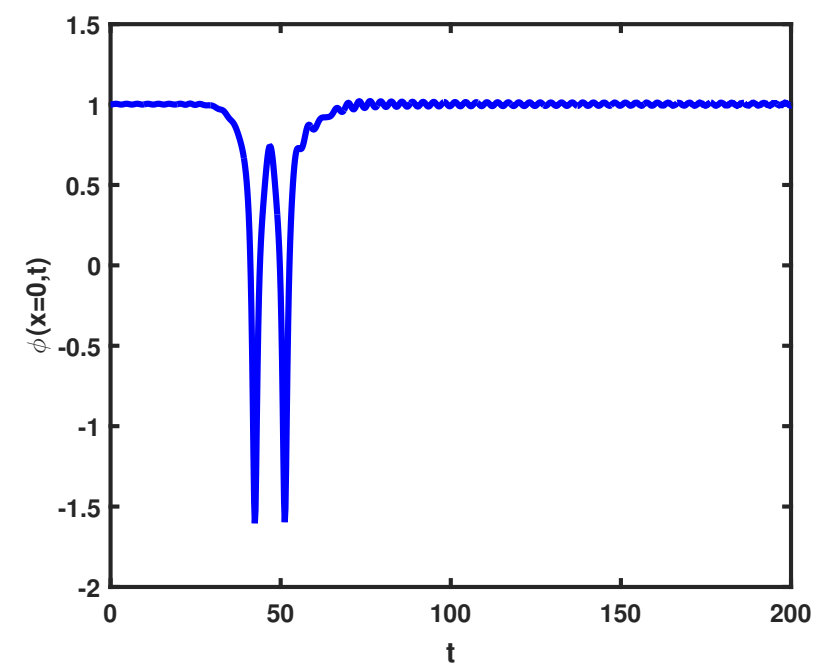

Figure 4. False vacuum in an unbounded interval: kink-antikink collision showing a zero order $(m=0)$ two-bounce for $\epsilon=0.01$ with $v=0.558$.

where $X$ is the translational collective coordinate. The total energy of the kink-antikink system for large values of $X$ is given by [82] $E_{K \bar{K}}=2 M_{K}-2(\Delta \rho) X$, where $M_{K}=2 \sqrt{2} M^{3} /(3 \lambda)$ is the classical kink mass and $\Delta \rho=\epsilon M^{4} / \lambda$ is the energy density difference between the two vacua. The energy is reduced for even larger values of $X$, meaning that soon after the scattering the kink-antikink pair separate at the velocity of light, as confirmed in figures $3 \mathrm{a}-\mathrm{c}$.

Another interesting aspect of kink-antikink collisions with the growing of $\epsilon$ is the appearing of a two-bounce window of zero order for $\epsilon \gtrsim 0.008$. An example of such effect is presented in figure 4 . There one can see that there is no oscillations of $\phi(x=0, t)$ between the bounces, meaning that $m=0$. The appearing of zero order two-bounce windows was reported before in models with degenerate vacua in the context of boundary scattering [66].

\subsection{False vacuum in a bounded interval: antikink-kink collisions}

We take as initial condition a $\phi^{4}$ antikink with velocity $v$ and a $\phi^{4}$ kink with velocity $-v$ :

$$
\begin{aligned}
& \phi(x, 0)=-\phi_{K}\left(x+x_{0}, v, 0\right)+\phi_{K}\left(x-x_{0},-v, 0\right)+1, \\
& \dot{\phi}(x, 0)=-\dot{\phi}_{K}\left(x+x_{0}, v, 0\right)+\dot{\phi}_{K}\left(x-x_{0},-v, 0\right) .
\end{aligned}
$$

The numerical procedure was the same used for kink-antikink collision. In this case, the attractive antikink-kink interaction and the tendency of the false vacuum to decay favor the confinement of the antikink-kink and its subsequent extinction. This can be explained using collective coordinates, following the same procedure described above [82] for kink-antikink scatering. The antikink-kink profile is aproximated by

$$
\phi(x) \approx \phi_{K}(x+X)-\phi_{K}(x-X)+\frac{M}{\sqrt{\lambda}} .
$$

For large values of $X$ the energy of the antikink-kink pair is given by $E_{\bar{K} K}=2 M_{K}+$ $2(\Delta \rho) X$. This means that energy favors the reduction of $X$, in a net confining effect. 

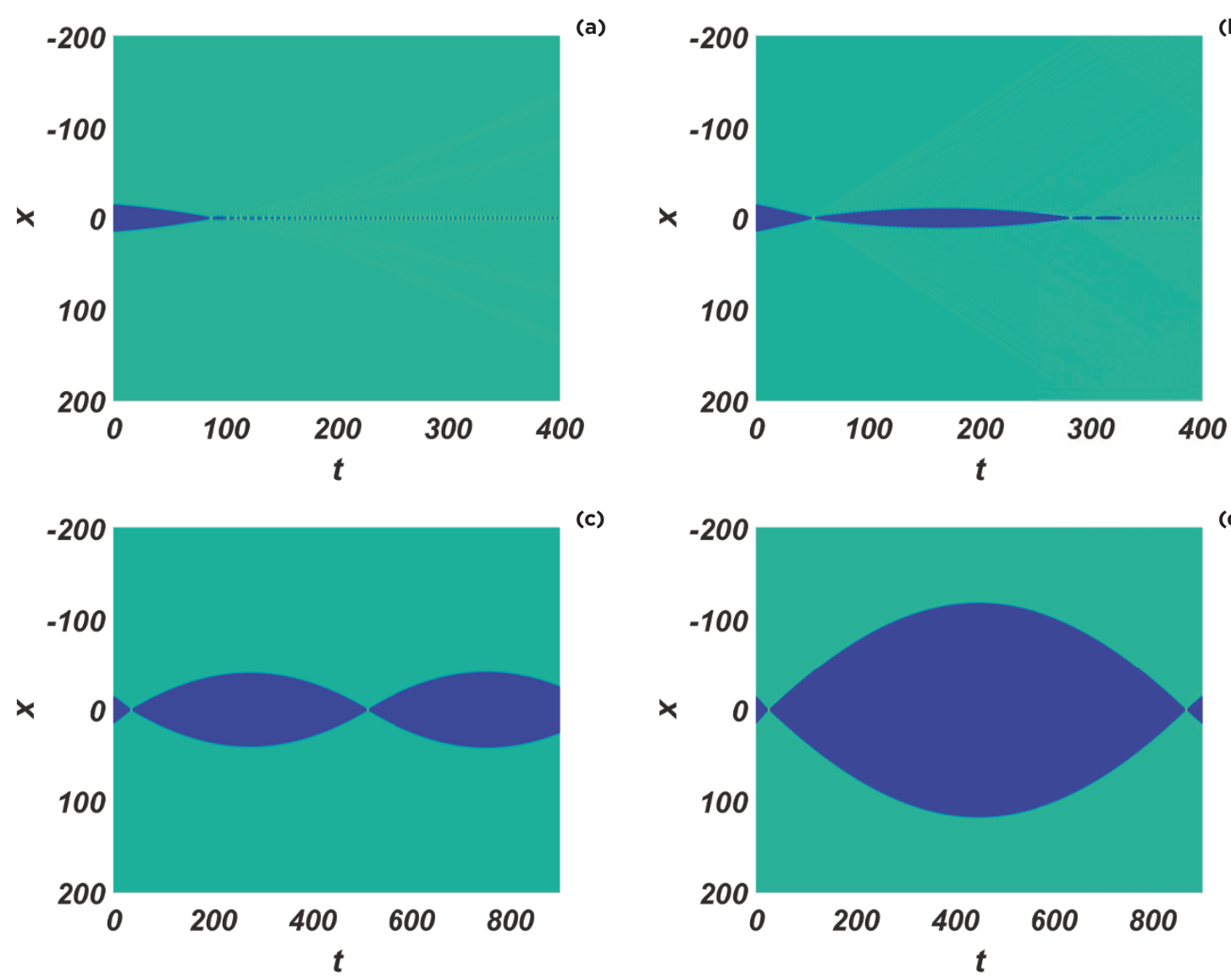

(c)

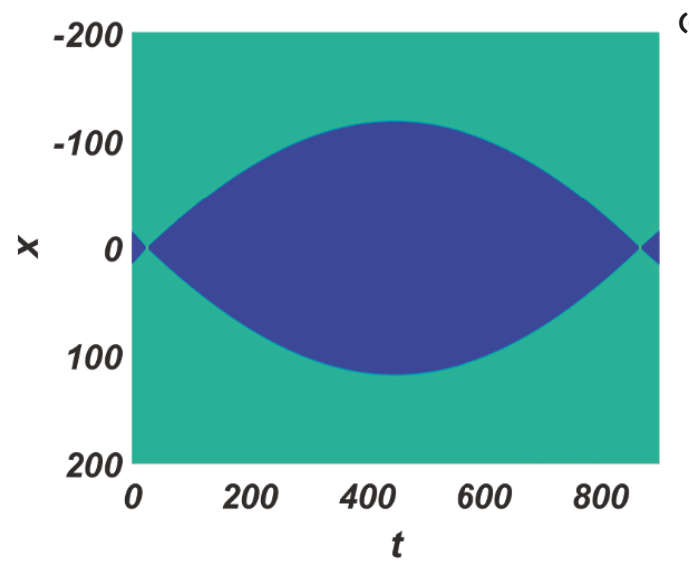

Figure 5. False vacuum in a bounded interval: antikink-kink collision for $\epsilon=0.001$ and with (a) $v=0.1$ (upper left), (b) $v=0.25$ (uper right), (c) $v=0.4$ (lower left), (d) $v=0.6$ (lower right). The figures show slices in the plane $(x, t)$ of the scalar field. Blue color represents the false vacuum $\phi=-1$ and the true vacuum $\phi=+1$ is represented in green.

The numerical simulations show that the complete process of vacuum decay has long duration and that the pattern of oscillations is intrincate. The output of mechanism is a two-step process: firstly the field bounces around the false vacuum with low frequency. Then the antikink-kink pair radiates and the field oscillates in a high-frequency, approaching in the long run to the true vacuum state. This is showed in figures $5 \mathrm{a}-5 \mathrm{~d}$ for $\epsilon=0.001$. Surprisingly larger initial velocities are not more effective in inducing the process of vacuum decay. On the contrary, with the growing of $v$ the low-frequency oscillation lasts even more before starting the decaying process of the false vacuum. It seems that, with false vacuum, larger initial velocities excite the vibrational state of the kink and antikink in a way that does not occurs for the degenerate vacuum, and the antikink-kink pair can bounce several times and be even more departed during such low-frequency oscillations (compare figures $5 \mathrm{a}-5 \mathrm{~b})$.

The relation between high- and low-frequency oscillations is more evident in a plot of the scalar field at the center of mass, $\phi(0, t)$, as shown in figures 6a-b. There one can 

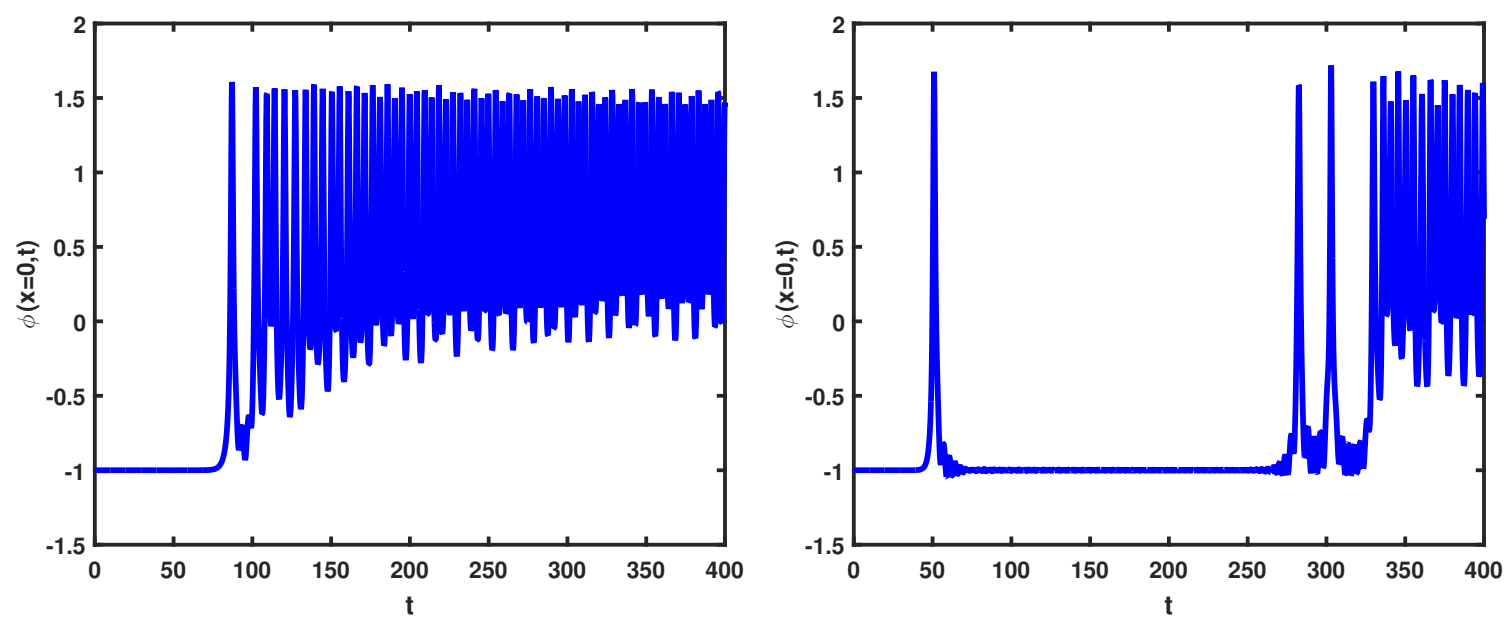

Figure 6. False vacuum in a bounded interval: antikink-kink collision. Scalar field at the center of mass, $\phi(0, t)$ as a function of time for $\epsilon=0.001$ with (a) $v=0.1$ and (b) $v=0.25$, corresponding to figures $5 \mathrm{a}-\mathrm{b}$.

see that, for a too low initial velocity, the field is not even able to bounce and the highfrequency process already takes place, as can be see in figure 6a for $v=0.1$. As an example for larger velocities we take $v=0.25$ as in figure $6 \mathrm{~b}$. There one can see three low-frequency oscillations around the false vacuum $\phi=-1$ before the field jumps and acquires the highfrequency oscillations that approach the true vacuum $\phi=+1$. As seen in figures $5 \mathrm{c}-5 \mathrm{~d}$, a too larger initial velocity would require a much longer running time of simulations to see the final high-frequency decay of the false vacuum. For the values $x_{0}=15$ considered in the simulations, the pattern of collisions described here is valid up to $\epsilon \sim 5 \times 10^{-3}$. Larger values of $\epsilon$ leads to a non-recognizable pattern, meaning that the nonlinear coupling for antikink-kink collisions is already too large.

An important issue is the possibility of oscillon formation connected to particle production due to vacuum decay. In an integrable theory, the particle and kink sectors are disconnected: that is, the kink-antikink pair scatter with at most a phase shift with no particle production. One knows that the integrable sine-Gordon model is dual to the massive Thirring model. In particular, the bound state of two massive Thirring fermions at strong coupling corresponds to a breather in the sine-Gordon model at small coupling [83]. Also, in the quantized breather to lowest order, the energy levels of the quantized breather corresponds to an integer number of particles, leading to the interpretation that the breather is a particle in the sine-Gordon model [84]. Then one expects that a breather-like profile could be a bridge between particle and soliton solutions in nonintegrable theories. This reasoning was used in the $\phi^{4}$ theory for the creation kink-antikink pairs and radiation after the scattering of small identical wave pulses [85]. The opposite process, that is, particle production in the wake of the colliding walls needs a better discussion of the breatheranalog for nonintegrable theories, the oscillon. The oscillon is an extremely long-lived state considered as a bound state of $\phi$-particles with a finite binding energy [86]. When quantized, the extremely stable classical oscillon solution has its lifetime shortened due to 

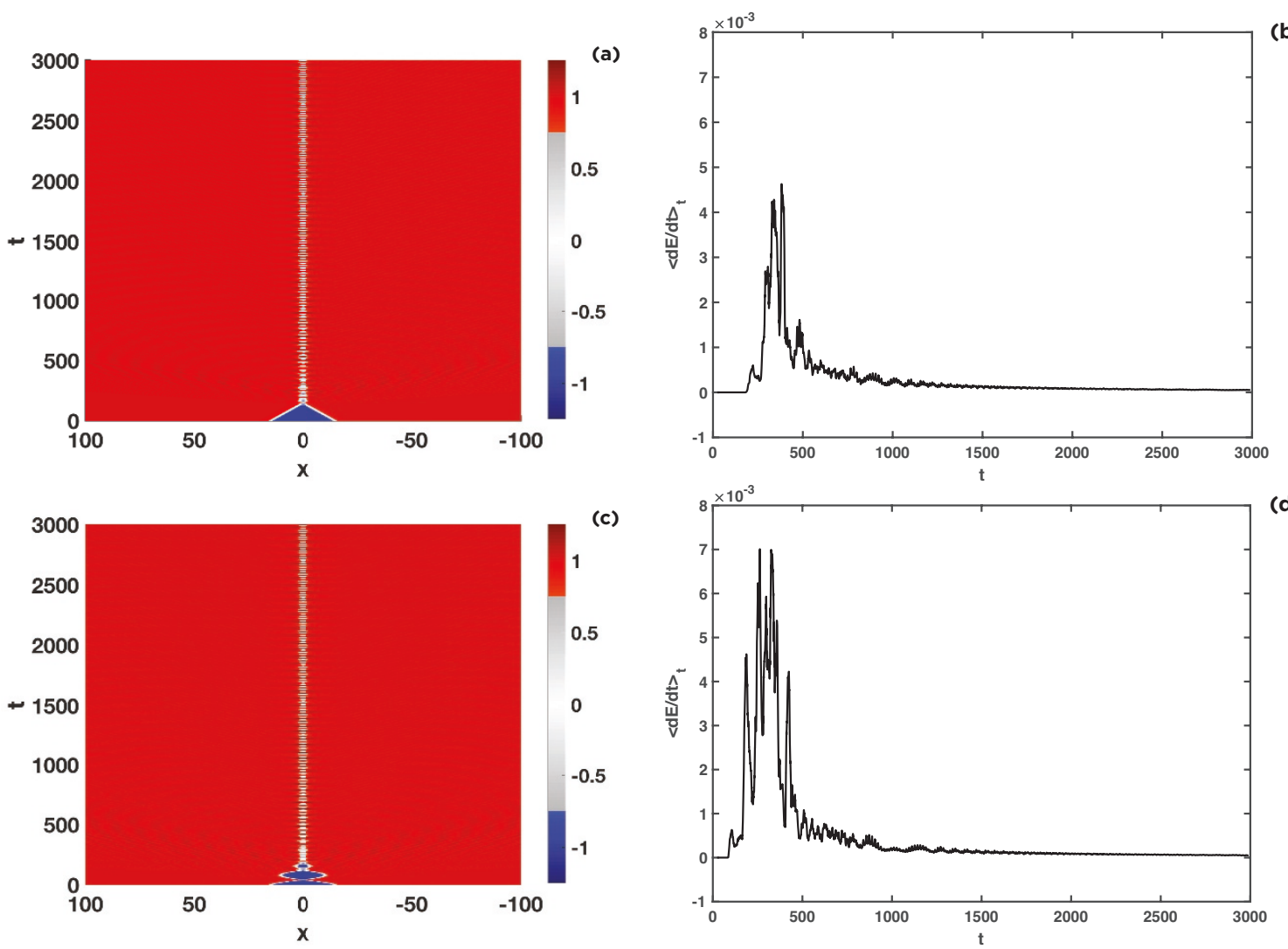

Figure 7. (Left) $\phi(x, t)$ showing long-lived oscillations after the scattering. (Right) Corresponding time-averaged energy-emission rate $\left\langle d E / d t>_{t}\right.$. The parameters chosed are $\epsilon=0$ (upper figures) and $\epsilon=10^{-2}$ (lower figures). In both cases we fixed $v=0.1$.

the emission of $\phi$-particles [87]. This means that the production of particles is connected in our classical setup by the production of oscillons. Our numerical simulations for the false vacuum in a bounded interval suggests the tendency of oscillon production in the long-term during the high-frequency oscillations of the scalar field at the center of mass, as showed in the figures $7 \mathrm{a}$ and $7 \mathrm{c}$. There one can see the long-lived oscillations after the scattering for several values of $\epsilon$.

The fact that the existence of false vacuum in a limited region induces energy dissipation is evident from the reduction of the maximum distance between antikink and kink with time, as shown for $\epsilon=0.01$ in figures $5 \mathrm{a}$ and $5 \mathrm{~b}$. The emission of radiation is quantitatively explained by the coupling of the vibrational (normal) mode with the states of the continuum. As a result of this coupling in nonlinear theories, an oscillating kink produces radiation with twice the frequency of the vibrational state. For example, for the kink of the $\phi^{4}$ model $(\epsilon=0)$, the rate of energy loss by the discrete mode, averaged over a period, is given by [88] $d E / d t \sim t^{-2}$, meaning an amplitude of the vibrational mode decaying as $A(t) \sim t^{-1 / 2}$. If the vibrational mode has low energy such that the second harmonic is bellow the continuum threshold, radiation is emitted by higher harmonics [66]. Once the oscillon is formed, the decay rate is considerably smaller, with [89] $d E / d t \sim \exp (-B / E)$. 
In general, even for $\epsilon \neq 0$, energy conservation results in the following expression for the energy density $\rho: d \rho / d t=\partial_{x}\left(\phi^{\prime} \dot{\phi}\right)$. Integrating the former expression in a box close to the scattered region and averaging in time results in the average emitted rate of energy (radiated power) $<d E / d t>_{t}$. The figures $7 \mathrm{~b}$ and $7 \mathrm{~d}$ present $<d E / d t>_{t}$ for several values of $\epsilon$. There one sees that radiation is emitted in bursts connected with the bouncings, and that the radiated power grows with $\epsilon$. We also verified that the radiated power grows for lower values of $\lambda$. After a transition, the decay rate is reduced considerably, but it is not suppressed, being very small with an almost constant rate for low energies, as expected for an oscillon. Despite the long-run oscillations described here still have not a single frequency (which characterize an oscillon in a strictly way), the behavior of low radiated power and long lifetime of the formed oscillatory state approaching the true vacuum with low amplitude, suggests the existence of a mechanism of oscillon formation.

We remark that we have confirmed with very good precision, for all cases considered, the conservation of total energy in the simulation box. For instance, when the false vacuum is in a limited region (antikink-kink collisions in our setup), and fixing $\lambda=1$, we have the following results before the bounces: for the case $\epsilon=0$, energy is conserved better than 1 part in $10^{9}$. The increasing of $\epsilon$ is acompanied with energy conservation with less precision. For the worst case analyzed, $\epsilon=0.01$, this means better 1 part in $10^{6}$. For the complete scattering process, for the case $\epsilon=0$, energy is conserved better than 1 part in $10^{7}$. For $\epsilon=0.01$, this means better than 1 part in $10^{4}$.

\section{Conclusions}

In this work we have studied the effect of kink scattering in the false vacuum decay. Kinkantikink collisions are characterized by two critical velocities, $v^{*}$ (not present for degenerate vacua) and $v_{\text {crit }}$. For $0<v<v^{*}$ we have inelastic scattering of the pair without contact. For $v^{*}<v<v_{\text {crit }}$ the scalar field at the center of mass $\phi(x, 0)$ can show bion or twobounce around the true vacuum. In a diagram in velocities we have the usual structure of bion states and a sequence of two-bounce windows accumulating around $v_{\text {crit }}$. For $\epsilon \gtrsim \bar{\epsilon}$ there is the appearance of zero-order two-bounce windows. For $v>v_{\text {crit }}$ we have inelastic scattering, and $\phi(x, 0)$ presents one-bounce around the true vacuum. Larger choices of initial position of the kink-antikink pair up to $x_{0}=25$ mean more time for the false vacuum to decay during the free propagation of the kink-antikink pair, leading to an increasing of $v^{*}$ and $v_{\text {crit. }}$.

It is relatively easier for a finite region in a true vacuum to expand through the false vacuum region, as described here in kink-antikink collisions. This is evident for low initial velocities, where the kink-antikink pair scatters at non-null distance. In comparison, it demands considerably more time for a finite region in false vacuum to decay completely to the true vacuum. We showed this for antikink-kink collisions. That is, the attractive kink-antikink interaction favors the formation of a long-lived bion state that inhibits the vacuum decay. The antikink-kink collisions were described in a two-step process with a low-frequency oscillation around the false vacuum followed by a high-frequency oscillation tending to the true vacuum in the long run. This two-step process resembles the behavior 
of what is known in the literature as false n-bounce-windows. In the end such bounce windows must be false since the scalar field will not remain in the initial state. We also investigated the effect of increasing the gap parameter $\epsilon$. For kink-antikink collisions we have the increasing of both velocities $v^{*}$ and $v_{\text {crit }}$.

For antikink-kink collisions the center of mass belongs to the false vacuum region and we have a complex mechanism for decay of the false vacuum: a low-frequency fast decaying bouncing state, followed by another long-lived high frequency bion state that radiates continuously, suggesting a transition in the long run to an oscillon state. For antikink-kink collisions, the more notable effect of the increasing of $\epsilon$ is the reduction of the maximum separation of the antikink-kink pair during the low-frequency oscillations. This shows the stronger tendency of the false vacuum to decay. The final state is the same, with the field oscillating fastly around the true vacuum and radiating energy in a low rate. We interpret that the radiation rate suffered a changing connected to a transition of energy from the vibrational state of the antikink-kink pair to an oscillon configuration. A smooth transformation from oscillational normal modes to oscillons was verified recently [90] in a modified $\phi^{4}$ theory with an additional potential that vanishes asymptotically. In the present work such transformation is induced by the false vacuum decay and occurs nonadiabatically. Depending on the initial velocity, the route to the oscillon formation is preceded by a twostep process, where sharp emission of radiation sincronized to the bouncings of the scalar field is folowed by a bion solution with an almost constant emission rate.

\section{Acknowledgments}

The authors thank FAPEMA - Fundação de Amparo à Pesquisa e ao Desenvolvimento do Maranhão through grants PRONEX 01452/14, PRONEM 01852/14, APCINTER00256/14, COOPI-07838/17, Universal-01061/17, Universal-01332/17, Universal-01191/16, and $\mathrm{CNPq}$ (brazilian agency) through grant 309842/2015-8 for financial support. This study was financed in part by the Coordenação de Aperfeiçoamento de Pessoal de Nível Superior - Brasil (CAPES) - Finance Code 001. P.P.A. work was supported by FEDER - Fundo Europeu de Desenvolvimento Regional funds through the COMPETE 2020 Operational Programme for Competitiveness and Internationalisation (POCI), and by Portuguese funds through FCT - Fundação para a Ciência e a Tecnologia in the framework of the project POCI-01-0145-FEDER-031938. P.P.A. also acknowledges the support provided by the FCT grant UID/FIS/04434/2013. 
Open Access. This article is distributed under the terms of the Creative Commons Attribution License (CC-BY 4.0), which permits any use, distribution and reproduction in any medium, provided the original author(s) and source are credited.

\section{References}

[1] J. García-Bellido, M. Garcia Perez and A. Gonzalez-Arroyo, Symmetry breaking and false vacuum decay after hybrid inflation, Phys. Rev. D 67 (2003) 103501 [hep-ph/0208228] [INSPIRE].

[2] H. Firouzjahi, S. Jazayeri, A. Karami and T. Rostami, Bubble nucleation and inflationary perturbations, JCAP 12 (2017) 029 [arXiv:1707.07550] [INSPIRE].

[3] R. Bousso, D. Harlow and L. Senatore, Inflation after False Vacuum Decay, Phys. Rev. D 91 (2015) 083527 [arXiv: 1309.4060] [INSPIRE].

[4] M. Dine, R.G. Leigh, P.Y. Huet, A.D. Linde and D.A. Linde, Towards the theory of the electroweak phase transition, Phys. Rev. D 46 (1992) 550 [hep-ph/9203203] [INSPIRE].

[5] G.W. Anderson and L.J. Hall, The Electroweak phase transition and baryogenesis, Phys. Rev. D 45 (1992) 2685 [INSPIRE].

[6] R. Apreda, M. Maggiore, A. Nicolis and A. Riotto, Gravitational waves from electroweak phase transitions, Nucl. Phys. B 631 (2002) 342 [gr-qc/0107033] [inSPIRE].

[7] A. Kobakhidze and A. Spencer-Smith, Electroweak Vacuum (In)Stability in an Inflationary Universe, Phys. Lett. B 722 (2013) 130 [arXiv:1301.2846] [INSPIRE].

[8] L. Leitao, A. Megevand and A.D. Sanchez, Gravitational waves from the electroweak phase transition, JCAP 10 (2012) 024 [arXiv:1205.3070] [INSPIRE].

[9] A. Kosowsky, M.S. Turner and R. Watkins, Gravitational radiation from colliding vacuum bubbles, Phys. Rev. D 45 (1992) 4514 [inSPIRE].

[10] E.J. Copeland, M. Gleiser and H.R. Muller, Oscillons: Resonant configurations during bubble collapse, Phys. Rev. D 52 (1995) 1920 [hep-ph/9503217] [INSPIRE].

[11] M. Kleban, Cosmic Bubble Collisions, Class. Quant. Grav. 28 (2011) 204008 [arXiv: 1107.2593] [INSPIRE].

[12] A. Aguirre and M.C. Johnson, A Status report on the observability of cosmic bubble collisions, Rept. Prog. Phys. 74 (2011) 074901 [arXiv: 0908.4105] [INSPIRE].

[13] J.R. Bond, J. Braden and L. Mersini-Houghton, Cosmic bubble and domain wall instabilities III: The role of oscillons in three-dimensional bubble collisions, JCAP 09 (2015) 004 [arXiv: 1505.02162] [INSPIRE].

[14] M.C. Johnson, C.L. Wainwright, A. Aguirre and H.V. Peiris, Simulating the Universe(s) III: Observables for the full bubble collision spacetime, JCAP 07 (2016) 020 [arXiv:1508.03641] [INSPIRE].

[15] R. Jinno, S. Lee, H. Seong and M. Takimoto, Gravitational waves from first-order phase transitions: Towards model separation by bubble nucleation rate, JCAP 11 (2017) 050 [arXiv: 1708.01253] [INSPIRE].

[16] A. Kusenko, P. Langacker and G. Segre, Phase transitions and vacuum tunneling into charge and color breaking minima in the MSSM, Phys. Rev. D 54 (1996) 5824 [hep-ph/9602414] [INSPIRE]. 
[17] G. Isidori, G. Ridolfi and A. Strumia, On the metastability of the standard model vacuum, Nucl. Phys. B 609 (2001) 387 [hep-ph/0104016] [INSPIRE].

[18] A. Rajantie and S. Stopyra, Standard Model vacuum decay with gravity, Phys. Rev. D 95 (2017) 025008 [arXiv:1606.00849] [INSPIRE].

[19] R.G. Landim and E. Abdalla, Metastable dark energy, Phys. Lett. B 764 (2017) 271 [arXiv:1611.00428] [INSPIRE].

[20] P.P. Avelino, C.J. A.P. Martins and L. Sousa, Dynamics of Biased Domain Walls and the Devaluation Mechanism, Phys. Rev. D 78 (2008) 043521 [arXiv:0805.4013] [InSPIRE].

[21] A.R. Bishop and T. Schneider eds., Solitons and Condensed Matter Physics, Springer-Verlag, Berlin (1978).

[22] Yu.S. Kivschar and G.P. Agrawal, Optical Solitons - From Fiber to Photonic Crystals, Academic Press, Burlington (2003).

[23] N. Manton and P. Sutcliffe, Topological Solitons, Cambridge University Press, Cambridge, England (2004).

[24] M.C. Birse, Soliton models for nuclear physics, Prog. Part. Nucl. Phys. 25 (1990) 1 [INSPIRE].

[25] A. Drago and V.M. Sarti, A chiral quark-soliton model with broken scale invariance for nuclear matter, Phys. Rev. C 86 (2012) 015211 [arXiv:1109.5399] [INSPIRE].

[26] S. Baldino, S. Bolognesi, S.B. Gudnason and D. Koksal, Solitonic approach to holographic nuclear physics, Phys. Rev. D 96 (2017) 034008 [arXiv:1703.08695] [INSPIRE].

[27] A. Vilenkin and E.P.S. Shellard, Cosmic Strings and Other Topological Defects, Cambridge University Press, Cambridge, England (1994).

[28] A. Aguirre and M.C. Johnson, A Status report on the observability of cosmic bubble collisions, Rept. Prog. Phys. 74 (2011) 074901 [arXiv:0908.4105] [inSPIRE].

[29] P.P. Avelino and R.P.L. Azevedo, Perfect fluid Lagrangian and its cosmological implications in theories of gravity with nonminimally coupled matter fields, Phys. Rev. D 97 (2018) 064018 [arXiv: 1802.04760] [INSPIRE].

[30] P.P. Avelino and L. Sousa, Matter Lagrangian of particles and fluids, Phys. Rev. D 97 (2018) 064019 [arXiv:1802.03961] [INSPIRE].

[31] T. Sugiyama, Kink-Antikink Collisions in the Two-Dimensional $\phi^{4}$ Model, Prog. Theor. Phys. 61 (1979) 1550 [INSPIRE].

[32] M. Moshir, Soliton-antisoliton scattering and capture in $\lambda \phi^{4}$ theory, Nucl. Phys. B 185 (1981) 318 [INSPIRE].

[33] D.K. Campbell, J.S. Schonfeld and C.A. Wingate, Resonance Structure in Kink-antikink interactions in $\phi^{4}$ theory, Physica D 9 (1983) 1 [INSPIRE].

[34] C.A. Wingate, Numerical Search for a $\phi^{4}$ Breather Mode, SIAM J. Appl. Math. 43 (1983) 120.

[35] D.K. Campbell, Solitary wave collisions revisited, Physica D 18 (1986) 47.

[36] T.I. Belova and A.E. Kudryavtsev, Quasi-periodic orbits in the scalar classical $\phi^{4}$ field theory, Physica D 32 (1988) 18. 
[37] P. Anninos, S. Oliveira and R.A. Matzner, Fractal structure in the scalar $\lambda\left(\phi^{2}-1\right)^{2}$ theory, Phys. Rev. D 44 (1991) 1147 [INSPIRE].

[38] R.H. Goodman and R. Haberman, Kink-Antikink Collisions in the $\phi^{4}$ Equation: The n-Bounce Resonance and the Separatrix Map, SIAM J. Appl. Dyn. Syst. 4 (2005) 1195.

[39] D. Saadatmand, S.V. Dmitriev, D.I. Borisov, P.G. Kevrekidis, M.A. Fatykhov and K. Javidan, Effect of the $\phi^{4}$ kink's internal mode at scattering on a PT-symmetric defect, Pisma Zh. Eksp. Teor. Fiz. 101 (2015) 550 [JETP Lett. 101 (2015) 497].

[40] P. Dorey and T. Romańczukiewicz, Resonant kink-antikink scattering through quasinormal modes, Phys. Lett. B 779 (2018) 117 [arXiv:1712.10235] [INSPIRE].

[41] P. Dorey, K. Mersh, T. Romanczukiewicz and Y. Shnir, Kink-antikink collisions in the $\phi^{6}$ model, Phys. Rev. Lett. 107 (2011) 091602 [arXiv:1101.5951] [INSPIRE].

[42] A. Demirkaya, R. Decker, P.G. Kevrekidis, I.C. Christov and A. Saxena, Kink dynamics in a parametric $\phi^{6}$ system: a model with controllably many internal modes, JHEP 12 (2017) 071 [arXiv: 1706.01193] [INSPIRE].

[43] V.A. Gani, A.E. Kudryavtsev and M.A. Lizunova, Kink interactions in the (1+1)-dimensional $\phi^{6}$ model, Phys. Rev. D 89 (2014) 125009 [arXiv:1402.5903] [INSPIRE].

[44] H. Weigel, Kink-Antikink Scattering in $\varphi^{4}$ and $\phi^{6}$ Models, J. Phys. Conf. Ser. 482 (2014) 012045 [arXiv: 1309.6607] [INSPIRE].

[45] T. Romańczukiewicz, Could the primordial radiation be responsible for vanishing of topological defects?, Phys. Lett. B 773 (2017) 295 [arXiv:1706.05192] [INSPIRE].

[46] E. Belendryasova and V.A. Gani, Resonance phenomena in the $\varphi^{8}$ kinks scattering, J. Phys. Conf. Ser. 934 (2017) 012059 [arXiv:1712.02846] [InSPIRE].

[47] V.A. Gani, V. Lensky and M.A. Lizunova, Kink excitation spectra in the (1+1)-dimensional $\varphi^{8}$ model, JHEP 08 (2015) 147 [arXiv:1506.02313] [INSPIRE].

[48] E. Belendryasova and V.A. Gani, Scattering of the $\varphi^{8}$ kinks with power-law asymptotics, arXiv: 1708.00403 [INSPIRE].

[49] M. Peyrard and D.K. Campbell, Kink-antikink interactions in a modified sine-Gordon model , Physica D 9 (1983) 33 [inSPIRE].

[50] V.A. Gani and A.E. Kudryavtsev, Kink-antikink interactions in the double sine-Gordon equation and the problem of resonance frequencies, Phys. Rev. E 60 (1999) 3305 [cond-mat/9809015] [INSPIRE].

[51] F.C. Simas, A.R. Gomes, K.Z. Nobrega and J.C. R.E. Oliveira, Suppression of two-bounce windows in kink-antikink collisions, JHEP 09 (2016) 104 [arXiv: 1605.05344] [INSPIRE].

[52] F.C. Simas, A.R. Gomes and K.Z. Nobrega, Degenerate vacua to vacuumless model and kink-antikink collisions, Phys. Lett. B 775 (2017) 290 [arXiv:1702.06927] [INSPIRE].

[53] V.A. Gani, A.M. Marjaneh, A. Askari, E. Belendryasova and D. Saadatmand, Scattering of the double sine-Gordon kinks, Eur. Phys. J. C 78 (2018) 345 [arXiv:1711.01918] [InSPIRE].

[54] D. Bazeia, E. Belendryasova and V.A. Gani, Scattering of kinks in a non-polynomial model, J. Phys. Conf. Ser. 934 (2017) 012032 [arXiv:1711.07788] [INSPIRE].

[55] D. Bazeia, E. Belendryasova and V.A. Gani, Scattering of kinks of the sinh-deformed $\varphi^{4}$ model, Eur. Phys. J. C 78 (2018) 340 [arXiv:1710.04993] [inSPIRE]. 
[56] R.H. Goodman and R. Haberman, Interaction of sine-gordon kinks with defects: the two-bounce resonance, Physica D 195 (2004) 303 [nlin/0310040].

[57] Z. Fei, Y.S. Kivshar and L. Vazquez, Resonant kink-impurity interactions in the sine-Gordon model, Phys. Rev. A 45 (1992) 6019.

[58] R.H. Goodman, P.J. Holmes and M.I. Weinstein, Interaction of sine-Gordon kinks with defects: phase space transport in a two-mode model, Physica D 161 (2002) 21.

[59] A. Halavanau, T. Romanczukiewicz and Ya. Shnir, Resonance structures in coupled two-component $\phi^{4}$ model, Phys. Rev. D 86 (2012) 085027 [arXiv:1206.4471] [InSPIRE].

[60] A. Alonso-Izquierdo, Reflection, transmutation, annihilation and resonance in two-component kink collisions, Phys. Rev. D 97 (2018) 045016 [arXiv:1711.10034] [INSPIRE].

[61] A. Alonso-Izquierdo, Kink dynamics in a system of two coupled scalar fields in two space-time dimensions, Physica D 365 (2018) 12 [arXiv:1711.08784] [INSPIRE].

[62] V.A. Gani, A.A. Kirillov and S.G. Rubin, Transitions between topologically non-trivial configurations, J. Phys. Conf. Ser. 934 (2017) 012046 [arXiv:1711.07700] [INSPIRE].

[63] A.M. Marjaneh, V.A. Gani, D. Saadatmand, S.V. Dmitriev and K. Javidan, Multi-kink collisions in the $\phi^{6}$ model, JHEP 07 (2017) 028 [arXiv: 1704.08353] [INSPIRE].

[64] A.M. Marjaneh, A. Askari, D. Saadatmand and S.V. Dmitriev, Extreme values of elastic strain and energy in sine-Gordon multi-kink collisions, Eur. Phys. J. B 91 (2018) 22 [arXiv: 1710.10159] [INSPIRE].

[65] D. Saadatmand, S.V. Dmitriev and P.G. Kevrekidis, High energy density in multisoliton collisions, Phys. Rev. D 92 (2015) 056005 [arXiv: 1506.01389] [InSPIRE].

[66] P. Dorey, A. Halavanau, J. Mercer, T. Romanczukiewicz and Y. Shnir, Boundary scattering in the $\phi^{4}$ model, JHEP 05 (2017) 107 [arXiv: 1508.02329] [INSPIRE].

[67] R. Arthur, P. Dorey and R. Parini, Breaking integrability at the boundary: the sine-Gordon model with Robin boundary conditions, J. Phys. A 49 (2016) 165205 [arXiv:1509.08448] [INSPIRE].

[68] A.R. Gomes, R. Menezes, K.Z. Nobrega and F.C. Simas, Kink-antikink collisions for twin models, Phys. Rev. D 90 (2014) 065022 [arXiv:1312.7519] [InSPIRE].

[69] L. Sousa and P.P. Avelino, p-brane dynamics in ( $N+1)$-dimensional FRW universes: a unified framework, Phys. Rev. D 83 (2011) 103507 [arXiv:1103.1381] [InSPIRE].

[70] J. Khoury, B.A. Ovrut, P.J. Steinhardt and N. Turok, The Ekpyrotic universe: Colliding branes and the origin of the hot big bang, Phys. Rev. D 64 (2001) 123522 [hep-th/0103239] [INSPIRE].

[71] Y.-i. Takamizu and K.-i. Maeda, Collision of domain walls and reheating of the brane universe, Phys. Rev. D 70 (2004) 123514 [hep-th/0406235] [INSPIRE].

[72] P.M. Saffin and A. Tranberg, Particle transfer in braneworld collisions, JHEP 08 (2007) 072 [arXiv:0705.3606] [INSPIRE].

[73] J. Omotani, P.M. Saffin and J. Louko, Colliding branes and big crunches, Phys. Rev. D 84 (2011) 063526 [arXiv:1107.3938] [INSPIRE].

[74] S.W. Hawking, I.G. Moss and J.M. Stewart, Bubble Collisions in the Very Early Universe, Phys. Rev. D 26 (1982) 2681 [INSPIRE]. 
[75] I.Yu. Kobzarev, L.B. Okun and M.B. Voloshin, Bubbles in Metastable Vacuum, Sov. J. Nucl. Phys. 20 (1975) 644 [INSPIRE].

[76] S.R. Coleman, The Fate of the False Vacuum. 1. Semiclassical Theory, Phys. Rev. D 15 (1977) 2929 [Erratum ibid. D 16 (1977) 1248] [INSPIRE].

[77] J. Braden, J.R. Bond and L. Mersini-Houghton, Cosmic bubble and domain wall instabilities I: parametric amplification of linear fluctuations, JCAP 03 (2015) 007 [arXiv:1412.5591] [INSPIRE].

[78] J. Braden, J.R. Bond and L. Mersini-Houghton, Cosmic bubble and domain wall instabilities II: Fracturing of colliding walls, JCAP 08 (2015) 048 [arXiv: 1505. 01857] [INSPIRE].

[79] V.G. Kiselev and Ya.M. Shnir, Forced topological nontrivial field configurations, Phys. Rev. D 57 (1998) 5174 [hep-th/9801001] [INSPIRE].

[80] E.B. Bogomolny, Stability of Classical Solutions, Sov. J. Nucl. Phys. 24 (1976) 449 [InSPIRE].

[81] M.K. Prasad and C.M. Sommerfield, An Exact Classical Solution for the 't Hooft Monopole and the Julia-Zee Dyon, Phys. Rev. Lett. 35 (1975) 760 [inSPIRE].

[82] S.J. Chang, Introduction to quantum field theory, World Scientific, Singapore (1990).

[83] S. Coleman, Aspects of Symmetry, Cambridge University Press, Cambridge (1985).

[84] T. Vashaspati, Kinks and Domain Walls: An Introduction to Classical and Quantum Solitons, Cambridge University Press, Cambridge (2006).

[85] S. Dutta, D.A. Steer and T. Vachaspati, Creating Kinks from Particles, Phys. Rev. Lett. 101 (2008) 121601 [arXiv:0803.0670] [INSPIRE].

[86] R.F. Dashen, B. Hasslacher and A. Neveu, The Particle Spectrum in Model Field Theories from Semiclassical Functional Integral Techniques, Phys. Rev. D 11 (1975) 3424 [InSPIRE].

[87] M.P. Hertzberg, Quantum Radiation of Oscillons, Phys. Rev. D 82 (2010) 045022 [arXiv: 1003.3459] [INSPIRE].

[88] N.S. Manton and H. Merabet, $\phi^{4}$ kinks: Gradient flow and dynamics, Nonlinearity 10 (1997) 3 [hep-th/9605038] [INSPIRE].

[89] G. Fodor, P. Forgacs, Z. Horvath and M. Mezei, Computation of the radiation amplitude of oscillons, Phys. Rev. D 79 (2009) 065002 [arXiv:0812.1919] [INSPIRE].

[90] T. Romańczukiewicz and Y. Shnir, Oscillons in the presence of external potential, JHEP 01 (2018) 101 [arXiv: 1706. 09234] [INSPIRE]. 